\title{
ISOMORPHISM TYPES IN WREATH PRODUCTS AND EFFECTIVE EMBEDDINGS OF PERIODIC GROUPS
}

\author{
BY \\ KENNETH K. HICKIN ${ }^{1}$ AND RICHARD E. PHILLIPS
}

\begin{abstract}
For any finitely generated group $Y, \omega(Y)$ denotes the Turing degree of the word problem of $Y$. Let $G$ be any non-Abelian 2-generator group and $B$ an infinite group generated by $k \geqslant 1$ elements. We prove that if $\tau$ is any Turing degree with $\tau \geqslant$ l.u.b. $\{\omega(G), \omega(B)\}$ then the unrestricted wreath product $W=G \operatorname{Wr} B$ has a $(k+1)$-generator subgroup $H$ with $\omega(H)=\tau$. If $B$ is also periodic, then $W$ has a $k$-generator subgroup $H$ such that $\tau=1$.u.b. $\{\omega(B), \omega(H)\}$.

Easy consequences include: $G \mathrm{Wr} \mathbf{Z}$ has $2^{\aleph_{0}}$ pairwise nonembeddable 2-generator subgroups and if $B$ is periodic then $G \mathrm{Wr} B$ has $2^{\aleph_{0}}$ pairwise nonembeddable $k$-generator subgroups.

Using similar methods, we prove an effective embedding theorem for embedding countable periodic groups in 2-generator periodic groups.
\end{abstract}

I. Introduction. Let $G$ denote a non-Abelian 2-generator group and $B$ an infinite group generated by $k \geqslant 1$ elements. $\mathbf{Z}$ is the infinite cyclic group. $G \mathrm{Wr} B=\Omega B=$ the unrestricted wreath product of $G$ and $B=$ the split extension of the Cartesian power $\Omega$ of $G$ over $B, \Omega=\{f \mid B \stackrel{f}{\rightarrow} G\}=$ the base group, by right $B$-translations of the domain, i.e. $f^{b}(c)=f\left(c b^{-1}\right)$ for all $b, c \in B$ and $f \in \Omega . \omega(B)$ is the Turing degree of the word problem of a group $B$. If $S \leqslant B$, then $\omega(S, B)$ is the Turing degree of the decision problem of $S$ in $B$, and the relation $\leqslant$ between Turing degrees is Turing reducibility.

Our first two theorems provide uniform means to obtain some old results and to prove some new ones on isomorphism types of finitely generated groups.

THEOREM 1. Let $\tau$ be any Turing degree such that $\tau \geqslant \omega(G \times B)$. Then:

(a) $G \mathrm{Wr} B$ has a $(k+1)$-generator subgroup $H$ satisfying $\omega(H)=\tau$;

(b) if $B$ is periodic, then $G \mathrm{Wr} B$ has a k-generator subgroup $H$ such that $\tau=$ $\omega(B \times H)$.

Note that the hypothesis that $G$ is non-Abelian is essential, at least if $B=\mathbf{Z}$, since there are only countably many isomorphism types of finitely generated metabelian groups (they are all recursively presented).

Note also that $\omega(G \times B)$ is the l.u.b. of $\omega(G)$ and $\omega(B)$ in the semilattice of Turing degrees. If $\tau$ and $\sigma$ are Turing degrees we let $\tau \times \sigma$ denote their l.u.b.

Received by the editors June 2, 1982.

1980 Mathematics Subject Classification. Primary 30E10, 20 F25.

Key words and phrases. Wreath product, nonembeddable finitely generated groups, periodic groups, effective embedding, word problem.

${ }^{1}$ Partly supported by NSF grant MCS 80-01777. 
While weaker than part (a), part (b) is strong enough to give the following general result.

COROLlaRY 1. (a) $G \mathrm{Wr} \mathbf{Z}$ has $2^{\aleph_{0}}$ pairwise nonembeddable 2-generator subgroups.

(b) If $B$ is periodic, then $G \mathrm{Wr} B$ has $2^{\aleph_{0}}$ pairwise nonembeddable k-generator subgroups.

Proof. Let $\sigma=\omega(G \times B)$. By [11, p. 12] there is a set of $2^{\aleph_{0}}$ Turing degrees $\left\{\tau_{\alpha} \mid \alpha<2^{\aleph_{0}}\right\}$ such that $\tau_{\alpha} \geqslant \sigma$ and $\tau_{\alpha} \neq \tau_{\beta}$ for $\alpha \neq \beta$ (that is, $\left\{\tau_{\alpha}\right\}$ is an antichain of $2^{\aleph_{0}}$ Turing degrees above $\sigma$ ). We apply Theorem $1(\mathrm{a})$ and (b) to obtain 2- (resp. $k$-) generator subgroups $H_{\alpha} \leqslant G \mathrm{Wr} B$ with $B=\mathbf{Z}$ in part (a) such that $\tau_{\alpha}=\sigma \times \omega\left(H_{\alpha}\right)$. If, for some $\alpha \neq \beta<2^{\aleph_{0}}, H_{\alpha}$ is isomorphic to a subgroup of $H_{\beta}$, then $\omega\left(H_{\alpha}\right) \leqslant \omega\left(H_{\beta}\right)$ implies $\tau_{\alpha}=\sigma \times \omega\left(H_{\alpha}\right) \leqslant \sigma \times \omega\left(H_{\beta}\right)=\tau_{\beta}$; a contradiction.

Corollary 2. Let $p$ be a Novikov-Adyan prime and let $B$ have exponent $p$ and be 2-generated. Then, for all $n \geqslant 3$, there are $2^{\aleph_{0}}$ pairwise nonembeddable 2-generator groups of exponent pn. Each of these groups $H$ has generators of orders $p$ and pn and $H$ has a class 2 nilpotent Hirsch-Plotkin radical $N$ of exponent $n$ with $H / N \cong B$.

Proof. We use part (b) of Corollary 1, choosing $G$ to be a class 2 nilpotent group of exponent $n$. The orders of the generators as well as the final properties will be evident in the proof of the theorem.

In the same way, we obtain

COROLlaRY 3. For every prime $p$ there are $2^{\aleph_{0}}$ pairwise nonembeddable 2-generator p-groups.

Other methods for obtaining $2^{\aleph_{0}}$ nonisomorphic 2-generator $p$-groups are known (e.g. [5, 6]). In [5], the method did not cover the cases $n=3,4$ or 5 of Corollary 2.

Let $H_{1}$ and $H_{2}$ be distinct groups as in Corollary 2. Thus $H_{i} / N_{i} \cong B$. Our construction does not yield $N_{1} \cong N_{2}$; although a detailed analysis of the construction for $B=\mathbf{Z}$ would disclose the possibility of obtaining mutual embeddability of $N_{1}$ and $N_{2}$. We leave as open questions whether $2^{\aleph_{0}} H$ exist as in Corollary 2 with isomorphic Hirsch-Plotkin radicals $N$, and whether any of the results here can be obtained for some periodic $B$ if $G$ is Abelian.

All of the subgroups in Theorem 1 are carefully prescribed analogues of those first used in [8] to obtain embedding theorems. The key to controlling the word problem in such subgroups is our Lemma 2 (see §II).

To prove Theorem 1 we need to construct special infinite subsets of $B$, similar to those used in [10], to serve as support sets of functions of $\Omega$. These are given in Lemma 1 (see §II) which also gives the computability conditions on these subsets needed in the proofs.

Philip Hall used a pigeonhole argument to produce $2^{\aleph_{0}}$ 2-generator center-bymetabelian groups with prescribed countable centers [2, p. 433]. Part (a) of Theorem 1 with $B=\mathbf{Z}$ does not yield center-by-metabelian groups, but certain 2-generator class 2 nilpotent-by-infinite cyclic groups

$$
H=\langle f, x\rangle \leqslant G \mathrm{Wr} \mathbf{Z}
$$


where $G$ is class 2 nilpotent, $\mathbf{Z}=\langle x\rangle$, and $f \in \Omega$. As we should expect, the isomorphism-types of those $H$ as in (1.1) can be distinguished much more dramatically than Theorem 1 suggests. Indeed, it is possible to determine $2^{\aleph_{0}} H$ 's as in (1.1) which are torsion-free and satisfy: the $2^{\aleph_{0}}$ classes $\mathfrak{K}_{H}=$ the nonmetabelian members of $Q S(H)$ (= sections of $H$ ) are pairwise disjoint. Further, an identical result can be obtained by elaborating Hall's construction to obtain center-by-metabelian groups with the above $Q S$-incomparability. These results will be presented in the forthcoming papers [4 and 13].

Hall's construction is the easiest and most elegant construction of $2^{\aleph_{0}}$ nonisomorphic 2-generator torsion-free groups of which we know. The standard method of proving this would be to use Britton's Lemma to embed every countable torsion-free group into a 2-generator torsion-free group having the same word problem degree (using the quick $H N N$ method [7, p. 188]). Though elementary, the use of Turing degrees is less so than Hall's construction. The use of Turing degrees does seem very appropriate to obtain nonembeddable groups.

The types of wreath embeddings used in Theorem 1 preserve word problems about as nicely as $H N N$ extensions do (see Lemma 2 in $\S \mathrm{II}$ ), and so can be used toward similar ends while producing groups of small exponent, etc. We will illustrate this by proving an effective embedding theorem similar to that using Britton's Lemma mentioned above, but for periodic groups.

Definition 1. Let $P=\langle X\rangle$ be a countable group where $X=\left\{x_{n} \mid n \geqslant 1\right\}$ is a recursive set of generators. Let $\tau$ be a Turing degree such that $\tau \geqslant \omega_{X}(P)=$ the Turing degree of the word problem of $P$ in the generators $X$ (if $P$ is finitely generated, then we need not specify $X$ and we write $\omega(P)$ ). Suppose $f: P \rightarrow G$ is an embedding of $P$ into a group $G=\langle Y\rangle$. We say that $f$ is $\tau$-effective provided:

(i) $\tau \geqslant \omega_{Y}(G)$

(ii) $\tau \geqslant \omega(f(P), G)$ (where $G$ is given in the generators $Y$ ); and

(iii) if $F$ is the free group with basis $Y$, then there is a subset $\left\{w_{n}\right\} \leqslant F$ which is recursive in $\tau$ such that in $G$ we have $f\left(x_{n}\right)=w_{n}$ for all $n \geqslant 1$.

If $\tau=\omega_{X}(P)$ we simply say that $f$ is an effective embedding of $P=\langle X\rangle$ into $G=\langle Y\rangle$. If $G$ is finitely generated, then we need not specify $Y$ since any finite generating set can be used.

It might be considered more natural to replace the condition "recursive in $\tau$ " in (iii) by the stronger condition "recursive" since the $\left\{w_{n}\right\}$ are merely a way to label the generators of $P$ in $G$, and not a decision procedure for them in $G$. However, the stronger condition that $\left\{w_{n}\right\} \leqslant F$ be recursive would present a rather serious roadblock to Theorem 2 below; hence we are forced to consider condition (iii) to be an appropriate one.

Note the following transitivity property of $\tau$-effectiveness: if $A \leqslant B \leqslant C$ are groups (with prescribed generating sets) and the embeddings $A \leqslant B$ and $B \leqslant C$ are $\tau$-effective, then $A \leqslant C$ is also $\tau$-effective.

It is well known that the groups of Golod [1] have recursively enumerable presentations. We do not know if finitely generated infinite $p$-groups exist which have solvable word problems, and hence we are forced to formulate Theorem 2 somewhat relativistically. 
THEOREM 2. Suppose $P=\langle X\rangle$ is a countably infinite, periodic $\pi$-group where $\pi$ is some set of primes and $\tau \geqslant \omega_{X}(P)$ is a Turing degree. Then, $P$ is $\tau$-effectively contained in a 2-generator periodic $\pi$-group if and only if there exists an infinite finitely generated periodic $\pi$-group whose word problem has Turing degree $\leqslant \tau$.

The forward implication is, of course, trivial. The construction of the embedding is a streamlined version of [10] where $P$ was embedded into a 2 -generator $\pi$-group but no attention was paid to recursiveness. The embedding has two major steps. The first is to embed $P$ into the derived group of a $\pi$-group $Q$. This can be done effectively in a standard manner via an unrestricted wreath product with the diagonal embedding, and we must also arrange in this step that $P$ is generated in a certain way by elements of bounded orders in $Q$. The second step embeds $Q^{\prime}$ in the 1-coordinate of a finitely generated subgroup of an unrestricted wreath product $Q \mathrm{Wr} B$ where $B$ is a certain finitely generated periodic $\pi$-group whose word problem has degree $\leqslant \tau$. The effectiveness of this embedding is similar to the method used in Theorem 1, the trick being to choose support sets effectively (see Lemma 2). Theorem 2 is proved in $\S \mathrm{V}$.

The extension of this effective embedding technique to residually finite groups will be undertaken in a forthcoming paper [3] where a careful analysis will be made of John Wilson's recent clever adaption of wreath embeddings to residually finite groups [12] in order to prove similar effective embedding theorems.

\section{Preliminaries.}

2.1. Here we state Lemma 1 which is needed for the proof of Theorem 1. The proof will be given in $\$ 2.2$.

LEMMA 1. Let $B=\langle D\rangle$ be an infinite group where $|D|=k$ and let $F$ be the free group with free basis $D$. Further, let $\left(w_{i} \mid i \geqslant 1\right)$ be some recursive enumeration of $F$, and $X$ be a fixed finite subgroup of $B$. Put $\beta=\omega(B)$. Then $B$ has an infinite subset $T=\left\{t_{n} \mid n \geqslant 1\right\}$ such that: $\beta$;

(i) $t_{1}=1$ and $\left(t_{n} \mid n \geqslant 1\right)$ is a nonredundant enumeration of $T$ which is effective in

(ii) $T \cap X=\{1\}$;

(iii) if $x \neq y \in X$, then $T x \cap T y=\varnothing$;

(iv) if $1 \in M \leqslant T$, then $T \cap\left(X M^{-1} M X\right)=M$;

(v) for all $1 \neq b \in B,|T \cap T b| \leqslant 2$.

If $b \in B$, let $\lambda(b)$ denote the least $j$ such that in $B$ we have $b=w_{j}$. Note that $\lambda$ is a $\beta$-computable function.

(vi) $T$ can be chosen so that for all $n \geqslant 1$ and $i<n$ we have

$$
\lambda\left(t_{n}^{-1} t_{i}\right) \geqslant n \text { and } \lambda\left(t_{i}^{-1} t_{n}\right) \geqslant n ;
$$

(vii) $\omega\left(T^{-1} T, B\right) \leqslant \beta$ and $\omega(T, B) \leqslant \beta$;

(viii) if $M \leqslant T$ and $\mu=\omega(M, B)$, then $\omega\left(M^{-1} M, B\right) \leqslant \beta \times \mu$;

(ix) there is a $\beta$-effective algorithm to compute the function $\sigma(b)=|T \cap T b|$ from $B$ to $\{0,1,2\}$ and (with $M$ as in (viii)) there is a $\beta \times \mu$-effective algorithm to compute the function $\sigma(b)=|M \cap M b|$ from $B$ to $\{0,1,2\}$. 
We first note that property (vii) is a consequence of (i) and (vi). To see this, let $b \in B$; to decide whether $b \in T^{-1} T$ we need only check, according to (vi), whether $b=t_{i}^{-1} t_{j}$ for some $1 \leqslant i, j \leqslant \lambda(b)$ and this can be done effectively in $\beta$ by the enumeration (i). Since $t_{1}=1$, this procedure also determines whether or not $b \in T$.

Likewise, (viii) follows from (i) and (vi); to specify an algorithm effective in $\mu \times \beta$ to decide membership in $M^{-1} M$ we may assume that $M$ is infinite. Let $b \in B$ be given; we first compute $\lambda(b)$ and begin generating the enumeration $\left\{t_{k(i)}\right\}$ of $M$. If $b \in M^{-1} M$, then by (vii) we must find that $t_{k(i)}^{-1} t_{k(j)}=b$ for some $\max (k(i), k(j)) \leqslant$ $\lambda(b)$; otherwise we will conclude that $b \notin M^{-1} M$.

Also (ix) follows from (i), (v), (vi) and (vii); for the members of $T \cap T b$ can be determined effectively in $\beta$ using (vi) and (vii) as follows. We first determine whether $b \in T^{-1} T$ and we compute $\lambda(b)$. If $b \notin T^{-1} T$, then $T \cap T b=\varnothing$; if $b \in T^{-1} T$, then by (vi) we need only check the values $t_{1}, \ldots, t_{\lambda(b)} \in T$ to find all pairs $s, t \in T$ such that $b=s^{-1} t$. Each such pair gives an element of $T \cap T b$ which we thus determine.

Hence, all parts of Lemma 1 follow if we establish parts (i)-(vi) and we take up the proof of these in the next section.

2.2. Proof of Lemma 1. The set $T$ will be constructed by induction. Let $t_{1}=1=w_{1}$; then obviously $T=\left\{t_{1}\right\}$ satisfies the conditions (i)-(vi). Suppose we have $\beta$-effectively (with a uniform algorithm) constructed $T_{k}=\left\{t_{1}, t_{2}, \ldots, t_{k}\right\}$ such that for $1 \leqslant n \leqslant k, T_{n}$ satisfies (i)-(vi) (in place of $T$ ). We will exhibit a uniform procedure effective in $\beta$ for selecting $t_{k+1} \in B$ such that $T_{k+1}$ satisfies (i)-(vi) and then define $T=\cup\left\{T_{k} \mid k \geqslant 1\right\}$.

For $1 \leqslant n \leqslant k$, let

$$
F_{n}=\left\{\prod_{i=1}^{4} a_{i} \mid a_{i} \in T_{n} \cup T_{n}^{-1} \cup X\right\} .
$$

Note that $F_{n}$ is finite and that $F_{i} \leqslant F_{j}$ when $1 \leqslant i \leqslant j \leqslant k$. We assume that during the construction we choose the $t$ 's so that

$$
t_{n} \notin F_{n-1} \text { for } 2 \leqslant n \leqslant k .
$$

Let $z$ be any element of $B$ with

$$
z \notin F_{k} \text {. }
$$

We now show

$$
T_{z}=T_{k} \cup\{z\}
$$

satisfies the properties (ii)-(v) of the lemma.

Parts (ii) and (iii) of (2.2.3) are proved by assuming that $T_{z}$ fails to satisfy the stated condition and then obtaining a contradiction of the form $z \in F_{k}$.

For the property (iv), let $1 \in M \leqslant T_{z}$ and note that $M \leqslant T_{z} \cap\left(X M^{-1} M X\right)$ follows from $1 \in T_{z} \cap M$. Now let $u \in T_{z} \cap\left(X M^{-1} M X\right)$; then there are elements $\alpha, \beta \in X$ and $v, w \in M$ such that

$$
u=\alpha v^{-1} w \beta .
$$


If $z$ does not occur in (2.2.4) the inductive hypothesis

$$
T_{k} \cap\left(\left(X\left(T_{k} \cap M\right)\left(T_{k} \cap M\right)^{-1} X\right)\right)=T_{k} \cap M
$$

gives $u \in M$. Thus, we assume that $z$ occurs in (2.2.4); further, since $z \notin F_{k}, z$ must occur exactly twice in (2.2.4). The cases $u=v=z$ or $u=w=z$ give the desired conclusion and we are left with the case $u=\alpha z^{-1} z \beta=\alpha \beta$ where $u \in T_{k}$. We now use (2.2.1) to deduce that $u=t_{1}=1 \in M$ and this gives property (iv).

To verify (v) for the set $T_{z}$ we suppose that for some $1 \neq d \in B$ we have $\left|T_{z} \cap T_{z} d\right| \geqslant 3$. Then there are three distinct elements $a, b, c$ of $T_{z}$ and elements $u, v, w$ of $T_{z}$ such that

$$
d=u^{-1} a=v^{-1} b=w^{-1} c .
$$

Evidently, $u, v, w$ are also distinct and our choice of $z$ forces $z$ to occur in each of the three products $u^{-1} a, v^{-1} b, w^{-1} c$; i.e., $z \in\{u, a\} \cap\{v, b\} \cap\{w, c\}$. This, in turn, contradicts the fact that $u, v$ and $w$ are distinct.

At this stage we have verified all parts of (2.2.3). Note that the property (2.2.2) is $\beta$-decidable since membership in $F_{k}$ is $\beta$-decidable.

Now let $U_{k}=\left\{w_{1}, \ldots, w_{k-1}\right\} \leqslant B$. Thus $R_{k}=T_{k} U_{k} \cup T_{k} U_{k}^{-1}$ is a finite subset of $B$. It is easy to show that $z \notin R_{k}$ implies (vi) of the lemma.

We now display the uniform $\beta$-effective algorithm for the choice of $t_{k+1}$. Using a $\beta$-oracle we check the elements $w_{i}$ of $B$ (with increasing subscripts) for

(1) membership in $B-F_{k}$, and

(2) membership in $B-R_{k}$.

The element $t_{k+1}$ is the first $w_{i}$ which meets these requirements; the existence of $t_{k+1}$ follows from the fact that both $R_{k}$ and $F_{k}$ are finite. We have already shown that $T_{k+1}=\left\{t_{1}, \ldots, t_{k}, t_{k+1}\right\}$ satisfies (ii)-(vi) and property (i) now also holds. This completes the proof of Lemma 1 .

2.3. The next lemma is the key for controlling Turing degrees. Necessary symbols and concepts are as in the introduction.

Lemma 2. Let $G=\langle X\rangle$ and $B$ be groups with $B$ finitely generated, let $f \in \Omega=$ the base group of $W=G \mathrm{Wr} B$ with $M=\operatorname{supp}(f)=\{b \in B \mid f(b) \neq 1\}$, and let $\tau$ be $a$ Turing degree. Assume the following three conditions.

(i) $\omega(B) \leqslant \tau$ and $\omega_{X}(G) \leqslant \tau$;

(ii) there is a $\tau$-effective algorithm for computing the values $f(b) \in\langle X\rangle(b \in B)$, and

(iii) for all $1 \neq c \in B, \sigma(c)=|M \cap M c|$ is finite and $\sigma(c)$ is computable by $a$ $\tau$-effective algorithm.

Then, there is a $\tau$-effective algorithm to decide, given $g \in f^{B} \leqslant\langle f, B\rangle \cap \Omega \leqslant W$, whether $\operatorname{supp}(g) \leqslant\{1\}$ and whether $g=1$.

Proof. Write $g=\left(f^{\varepsilon_{1}}\right)^{b_{1}} \cdots\left(f^{\varepsilon_{n}}\right)^{b_{n}}$ where, for $1 \leqslant i \leqslant n, \varepsilon_{i}= \pm 1$ and $b_{i} \in B$. For $1 \leqslant i \leqslant n$, compute the exponent sum

$$
\mu_{i}=\sum_{1 \leqslant j \leqslant n}\left\{\varepsilon_{j} \mid b_{j}=b_{i}\right\}
$$


using an oracle for $\tau \geqslant \omega(B)$. Define

$$
F_{1}=\bigcup\left\{M b_{i} \cap M b_{j} \mid 1 \leqslant i, j \leqslant n \text { and } b_{i} \neq b_{j}\right\}
$$

and note that $F_{1}$ is finite by (iii).

Suppose first that, for all finite subsets $M_{0} \leqslant M,\left\{|f(s)| \mid s \in M-M_{0}\right\}$ is unbounded. If $\mu_{i} \neq 0$ for some $i$, then we claim that $\operatorname{supp}(g)$ is infinite because we can choose infinitely many $x \in M b_{i}-F_{1}$ such that $\left|f\left(x b_{i}^{-1}\right)\right|$ does not divide $\mu_{i}$ and we have $g(x)=\left[f^{b_{i}}(x)\right]^{\mu_{i}}=f\left(x b_{i}^{-1}\right)^{\mu_{i}} \neq 1$.

On the other hand suppose that there is a bound on almost all of the numbers $|f(x)|, s \in M$. Define for $d>1 U_{d}=\{b \in M|| f(b) \mid=d\}$ and let $r=$ least common multiple of all those $d$ 's for which $U_{d}$ is infinite; thus, $r$ is finite by the hypothesis of this case. If, for some $i, r$ does not divide $\mu_{i}$, then there is some $d>1$ such that $U_{d}$ is infinite and $d$ does not divide $\mu_{i}$ and an argument identical to the first case above shows that $\operatorname{supp}(g)$ is infinite.

Thus, in these two cases we would decide that $\operatorname{supp}(g) \$\{1\}$ and so we may suppose either:

Case A. $\mu_{i}=0$ for all $1 \leqslant i \leqslant n$, or

Case B. There is a finite subset $V \leqslant M$ and for all $s \in M-V,|f(s)|$ divides $r$ (as defined above) and $r$ divides $\mu_{i}$ for all $1 \leqslant i \leqslant n$.

Note that since $f$ is given we determine whether Case $\mathbf{A}$ or $\mathbf{B}$ holds after computing the $\mu_{i}$. Define $F_{2}=\varnothing$ if Case A holds; and $F_{2}=V b_{1} \cup \cdots \cup V b_{n}$ if Case A does not hold, but Case B holds. Thus $F_{1} \cup F_{2}$ is finite and an easy argument shows that $\operatorname{supp}(g) \leqslant F_{1} \cup F_{2}$. From the hypotheses (i) and (ii), the lemma will be proved if we can display a $\tau$-effective algorithm for generating (in a definite finite time) a complete list of $F_{1} \cup F_{2}$. Since $V$ can be specified as soon as $f$ is known, there is no problem in constructing a list of $F_{2}$. To list $F_{1}$ we observe

$$
z \in F_{1} \text { if and only if there exist } u, v \in M \text { and } b_{i} \neq b_{j} \text { such that } z=u b_{i}=v b_{j} .
$$

Thus, to effectively list (with $\tau$-oracle) all $z \in F_{1}$, we first decide for each pair $b_{i} \neq b_{j}$ whether $\sigma\left(b_{i} b_{j}^{-1}\right) \neq 0$, i.e. whether $M b_{i} \cap M b_{j} \neq \varnothing$, by computing this value of $\sigma$ in accordance with (iii). If we find $\sigma\left(b_{i} b_{j}^{-1}\right) \neq 0$, then we begin generating a $\tau$-effective enumeration of $M=\operatorname{supp}(f)$ (this can be done by (i) and (ii)) and listing all pairs $u, v \in M$ (as we find them) for which $b_{i} b_{j}^{-1}=u^{-1} v$. Now each such distinct pair corresponds to a distinct element of $M b_{i} \cap M b_{j}$, and hence we must be able to list exactly $\sigma\left(b_{i} b_{j}^{-1}\right)=\left|M b_{i} \cap M b_{j}\right|$ such pairs. Since $\sigma$ is a $\tau$-computable function on $B$, we will know to stop the enumeration when exactly $\sigma\left(b_{i} b_{j}^{-1}\right)$ distinct pairs $(u, v)$ have been found. These pairs $(u, v)$ (for all possible $b_{i} \neq b_{j}$ ) give us an exhaustive list of $F_{1}$ by (2.3.1).

We will also need a simple lemma on relative recursiveness.

Lemma 3. If $T$ is an infinite set of Turing degree $\leqslant \beta$ and $\tau \geqslant \beta$ is a Turing degree, then there is an infinite subset $M$ of $T$ whose Turing degree $\mu$ satisfies $\tau=\mu \times \beta$. 
Proof. Let $T=\left\{t_{k} \mid k \geqslant 1\right\}$ and let $S=\left\{k_{i} \mid i \geqslant 1\right\}$ be an infinite set of natural numbers of Turing degree $\tau$. Define $M=\left\{t_{k_{i}} \mid i \geqslant 1\right\}$. One checks easily that the Turing degree $\mu$ of $M$ satisfies $\tau=\mu \times \beta$.

III. Proof of Theorem 1(a). We first define elements in the base group of $W=$ $G \mathrm{Wr} B=\Omega B$ which have special support sets and which are used in the proofs of both parts of Theorem 1 .

Let $M$ be an infinite subset of $T$ with $1 \in M$ where $T \leqslant B$ is the subset given in Lemma 1. Let $G=\langle x, y\rangle$. Define $f=f_{M} \in \Omega$ as follows.

$$
f_{M}(1)=x, f_{M}(t)=y \quad \text { if } t \in M-\{1\} \quad \text { and } \quad f_{M}(u)=1 \quad \text { if } u \notin M .
$$

For the proof of part (a) of Theorem 1, we define

$$
H=H_{M}=\langle f, B\rangle \leqslant W
$$

and note that $H$ has $k+1$ generators.

Let $\tau \geqslant \omega(G \times B)$ be a Turing degree. From Lemma 3 we obtain $M \leqslant T$ satisfying

$$
\tau=\omega(M, B) \times \omega(B) .
$$

Since the addition of 1 to $M$ will leave (3.3) unchanged, we can assume $1 \in M$.

We must show that $\omega(H)=\tau$.

To show that $\omega(H) \leqslant \tau$ suppose that $w$ is a word in the generators of $H$. We first write $w$ in the form $b h$ where $h \in \Omega$ is a product of conjugates of $f^{ \pm 1}$ and $b \in B$. Since $\omega(B) \leqslant \tau$ we decide $\tau$-effectively whether $b=1$. If $b \neq 1$, then $w \neq 1$; so assume we find $b=1$. We now use Lemma 2 to decide $\tau$-effectively whether $w=h=1$. Hypothesis (i) of Lemma 2 holds, i.e. $\tau \geqslant \omega(G \times B)$, (ii) holds by (3.1) and (3.3), and (iii) holds according to (ix) of Lemma 1.

To establish $\tau \leqslant \omega(H)$, assume that a decision procedure for $\omega(H)$ is available. Since the $k$ generators of the top group $B$ of $W$ are among the generators of $H$, we can decide $\omega(B)$. In view of (3.3) we need only display a decision procedure for $\omega(M, B)$ to complete our proof. Let

$$
\Delta_{M}=\left\{b \in B \mid\left[f^{b}, f\right] \neq 1\right\} .
$$

It is not difficult to show, using (3.1) that $\Delta_{M}=(M-\{1\}) \cup\left(M^{-1}-\{1\}\right)$. Hence this set is $\omega(H)$-decidable.

Since we have a decision procedure for $\omega(B)$ available, we can also decide the set $M \cup M^{-1}$. Since $M \cup M^{-1} \leqslant M^{-1} M$ (because $\left.1 \in M\right)$, we have $T \cap\left(M \cup M^{-1}\right)=$ $M$ by (iv) of Lemma 1 . Since $\omega(T, B) \leqslant \omega(B)$, the above intersection property provides a decision procedure for $\omega(M, B)$ which is effective in $\omega(H)$, thus finishing the proof.

IV. Proof of Theorem 1(b). We will denote any sequence $\left(b_{1}, \ldots, b_{k-1}\right)$ having $k-1$ terms by $\vec{b}$. Let $F$ be a free group of rank $k$ with free generators $\{s, \vec{t}\}$ and $B=\langle a, \vec{b}\rangle$. Let $F=\left(w_{n} \mid n \geqslant 1\right)$ be a recursive list of $F$ where $i \leqslant j$ implies that the length of $w_{i}$ is less than or equal to the length of $w_{j}$. 
Let $\theta$ be the homomorphism from $F$ onto $B$ defined by $\theta(s)=a$ and $\theta(\vec{t})=\vec{b}$. Then with $\lambda$ defined as in Lemma 1 and $b \in B$ we have

(4.1) if $\lambda(b)=j$, then $w_{j}$ is a word of minimal length in the coset $\theta^{-1}(b)$. Thus,

(4.2) $\mathscr{B}=\left\{w_{j} \mid j=\lambda(b)\right.$ for some $\left.b \in B\right\}$ is a transversal of $\operatorname{ker} \theta$ in $F$ with $1 \in \Re$.

We also adopt the notation

(4.3) if $U=\langle r, \vec{v}\rangle$ is a k-generator group and $w \in F$, then $w(r, \vec{v})$ is the image of $w$ in $U$ under the unique homomorphism of $F$ to $U$ that maps $s$ to $r$ and $\vec{t} t ? \vec{v}$. In the special case $U=B=\langle a, \vec{b}\rangle$ we use the notation $w(a, \vec{b})=w^{0}$.

Recalling that $B$ is periodic, let $n=|a|, X=\langle a\rangle$, and let $T \leqslant B$ be the subset given in Lemma 1 (for our particular choice of $X \leqslant B$ and $\lambda$ ). Let $\tau \geqslant \omega(B \times G)$ be a Turing degree and choose $M \leqslant T$ as in (3.3) and so that $1 \in M$. We define $f=f_{M} \in \Omega$ as in (3.1). Define

$$
H=H_{M}=\left\langle a f, b_{1}, \ldots, b_{k-1}\right\rangle,
$$

and

$$
g=g_{M}=(a f)^{n}=f^{a^{n-1}} \cdots f^{a} f \quad(\text { since } n=|a|) .
$$

Thus, $g \in H \cap \Omega$ and property (iii) of Lemma 1 together with (3.1) gives

$$
\operatorname{supp}(g)=M X \text { and } g(c)=x \text { if } c \in X \quad \text { while } g(c)=y \text { if } c \in M X-X .
$$

Note that if $h \in \Omega$ and $w \in B$, then

(4.7) $w(a h, \vec{b})=w^{0}\left(h^{\varepsilon_{1}}\right)^{w_{1}^{0}} \cdots\left(h^{\varepsilon_{n}}\right)^{w_{n}^{0}}$, where for $1 \leqslant i \leqslant n, \varepsilon_{i}= \pm 1$ and $w_{i}$ is a proper final segment of $w$.

An easy consequence of (4.1) and (4.2) is the crucial fact

(4.8) if $1 \neq w \in \mathscr{B}$ and $w_{1}, \ldots, w_{n}$ are proper final segments of $w$, then

$$
\left(f_{M}^{\varepsilon_{1}}\right)^{w_{1}^{0}} \cdots\left(f_{M}^{\varepsilon_{n}}\right)^{w_{n}^{0}}\left(w^{0}\right) \in\langle y\rangle .
$$

To prove (4.8) note that the choice of our set $\mathscr{B}\left((4.1)\right.$ and (4.2)) forces $w^{0}\left(w_{i}^{0}\right)^{-1} \neq 1$ for $1 \leqslant i \leqslant n$.

Thus, for each $i, f_{M}^{\varepsilon}\left(w^{0}\left(w_{i}^{0}\right)^{-1}\right)$ is either 1, $y$ or $y^{-1}$ and (4.8) follows easily.

Now define

$$
\Delta_{M}=\left\{w \in \mathscr{B} \|\left[g^{w(a f, \vec{b})}, g\right] \neq 1\right\} \cup\{1\}
$$

and note the slight technical difference from the way $\Delta_{M}$ was defined in $\S I I I$. The key observation is

LEMMA 4. $M \leqslant \Delta_{M}^{0} \leqslant X M^{-1} M X$.

Proof. It follows from (4.6) and (iii) of Lemma 1 that $g_{M}(1)=x$ and that $g_{M}\left(w^{0}\right)=y$ if $1 \neq w^{0} \in M$. Let $1 \neq w^{0} \in M$; then, as in (4.7),

$$
w\left(a f_{M}, \vec{b}\right)=w^{0} h, \quad \text { where } h=\left(f_{M}^{\varepsilon_{1}}\right)^{w_{1}^{0}} \cdots\left(f_{M}^{\varepsilon_{n}}\right)^{w_{n}^{0}} .
$$


Thus (4.8) implies that $h\left(w^{0}\right) \in\langle y\rangle$, and so

$$
\begin{aligned}
{\left[g_{M}^{w\left(a f_{M}, \vec{b}\right)}, g_{M}\right]\left(w^{0}\right) } & =\left[g_{M}^{w^{0} h}, g_{M}\right]\left(w^{0}\right)=\left[\left(g_{M}\left(w^{0}\left(w^{0}\right)^{-1}\right)\right)^{h\left(w^{0}\right)}, g_{M}\left(w^{0}\right)\right] \\
& =\left[g_{M}(1)^{h\left(w^{0}\right)}, g_{M}\left(w^{0}\right)\right]=\left[x^{y^{i}}, y\right],
\end{aligned}
$$

which is not 1 since $[x, y] \neq 1$. Hence $w^{0} \in \Delta_{M}^{0}$.

Suppose now that $w \in \Delta_{M}$; then $\left[g_{M}^{w\left(a f_{M}, \vec{b}\right)}, g_{M}\right] \neq 1$ and using the notation above, $\left[g_{M}^{w^{0} h}, g_{M}\right] \neq 1$. Now by (3.9) and standard facts about wreath products, $\operatorname{supp}\left[g_{M}^{w^{0} h}, g_{M}\right] \leqslant M X w^{0} \cap M X$ and so $M X w^{0} \cap M X \neq 1$. Thus, $w^{0} \in X M^{-1} M X$ and this completes the proof of Lemma 4.

Proof that $\omega(B \times H) \leqslant \tau$. We may assume that we have available decision procedures for $M$, for $T$, and for the word problem of $B$, and we want to construct a decision procedure for the word problem of $H$. Let

$$
d=w(a f, \vec{b})=w^{0}\left(f^{\varepsilon_{n}}\right)^{w_{1}^{0}} \cdots\left(f^{\varepsilon_{n}}\right)^{w_{n}^{0}}
$$

be an element of $H$ as expressed in (4.7). We first decide whether $w^{0}=w(a, \vec{b})=1$ in $B$. We may assume that we find $w^{0}=1$ for otherwise $d \neq 1$. Thus $d=\left(f^{\varepsilon_{1}}\right)^{w_{1}^{0}}$ $\cdots\left(f^{\varepsilon_{n}}\right)^{w_{n}^{0}}$.

Since $\tau \geqslant \omega(G \times B) \times \omega(M, B)$ and in view of (3.1) and (ix) of Lemma 1, all hypotheses of Lemma 2 are satisfied and Lemma 2 gives a $\tau$-effective algorithm to decide whether $d=1$.

Proof that $\tau \leqslant \omega(B \times H)$. We assume that we have available oracles for $\omega(B)$ and $\omega(H)$. We will first observe that this enables us to decide $\omega\left(\Delta_{M}^{0}, B\right)$. Suppose $b \in B$. Using our $\omega(B)$-oracle we can determine the unique $w \in \mathscr{B}$ such that $b=w^{0}$. Thus, to decide whether $b \in \Delta_{M}^{0}$, consulting (4.9), it suffices to decide whether either $b=1$ or $\left[g^{w(a f, b)}, g\right] \neq 1 \in H$ which we can do with our oracles. Now, in view of the equation $T \cap X M^{-1} M X=M$ ((iv) of Lemma 1) and Lemma 4, we have $T \cap \Delta_{M}^{0}=M$ and hence $\omega(M, B)$ can be decided with our oracles for $\omega(B)$ and $\omega(H)$; and in view of (3.3) we can also decide $\tau$.

V. Proof of Theorem 2.

5.1. We first note some elementary facts regarding restricted wreath products.

(5.1.1) Let $H=\left\langle h_{1}, \ldots, h_{n}\right\rangle$ be a finitely generated group and put $B=H \mathrm{wr} H$. Then $B$ has generating set $\left\{h_{1}, \ldots, h_{n}, \bar{h}_{1}, \ldots, \bar{h}_{n}\right\}$ where for $1 \leqslant i \leqslant n, \bar{h}_{i}$ is the function in the base group of $B$ with $\operatorname{supp}\left(\bar{h}_{i}\right)=\{1\}$ and $\bar{h}_{i}(1)=h_{i}$. Further, $\omega(B)=$ $\omega(H)$.

The equality $\omega(B)=\omega(H)$ follows from the fact that elements $x \in B$ have the unique representation $x=h c$ where $h \in H$ and $c$ is in the base group of $B$ and both $h$ and $c$ are $\omega(H)$-computable.

(5.1.2) Let $f$ be an element in the base group of $B$ with $f^{2} \neq 1$ and $\operatorname{supp}(f)=\{1\}$. Then the set $M=\left\{f^{h} \mid h \in H\right\}$ has the properties

(i) for all $1 \neq c \in B$, the equation $c=u^{-1} v$ can hold for at most one pair $(u, v) \in M \times M$; this is called sparseness property [5, p. 181]; and is equivalent to $|M \cap M c| \leqslant 1$ if $1 \neq c \in B$; 
(ii) $\omega(M, B) \times \omega\left(M^{-1} M, B\right) \leqslant \omega(H)$;

(iii) for all $1 \neq c \in B, \sigma(c)=|M \cap M c|$ is computable by an $\omega(H)$-effective algorithm.

In (5.1.2), the verification of (i) is straightforward while (ii) follows as in the proof of (5.1.1). For (iii), we see from (i) that $|M \cap M c| \leqslant 1$. Note that $|M \cap M c|=1$ if and only if $c \in M^{-1} M$ and this can be decided by (ii). Thus (iii) holds, and we note that

(5.1.3) if $\tau \geqslant \omega(H), M$ satisfies the condition (iii) of Lemma 2.

It is also convenient to record here a restricted version of Theorem 2.

LEMMA 5. If $R$ is a finitely generated periodic $\pi$-group, then $R$ is effectively contained in a 2-generator periodic $\pi$-group $S$.

Proof. The embedding is the one given first by Neumann and Neumann [8] (see also Phillips [10, p. 251]) and the reader is referred to these proofs. More complicated variants of this procedure will appear in the proof of Theorem 2; here we only give a very brief sketch of the embedding. The essential features are given in

(5.1.4) there are finite cyclic groups $C_{n}$ and $C_{k}$ and a function $f$ in the base group of ( $R$ wr $C_{n}$ ) wr $C_{k}$ such that $R$ is embedded in $f^{C} k \leqslant\left\langle f, C_{k}\right\rangle$ as the diagonal of the 1-coordinate subgroup.

The effectiveness of this embedding follows easily from the fact that both $C_{n}$ and $C_{k}$ are finite.

5.2. The construction. Let $P=\langle X\rangle$ be an infinite periodic $\pi$-group and $p$ be a fixed prime involved in $P$. Put

$$
n= \begin{cases}p & \text { if } p \text { is odd } \\ 4 & \text { if } p=2\end{cases}
$$

There are two steps in the embedding of Theorem 2.

Step 1 . We will embed $P$ effectively into a periodic $\pi$-group $Q=\langle Y\rangle$ in such a way as to satisfy:

(5.2.1) For each $x \in X$ there are elements $c_{1}, c_{2}, c_{3}, c_{4}$ in $Q$ such that $x=\left[c_{1} c_{2}, c_{3} c_{4}\right]$ and the $c_{i}$ 's may be chosen so that $c_{i}=\left[d_{i}, e_{i}\right]$ where $d_{i}$ and $e_{i}$ are elements of $Q$ of order $n$ which are $\omega_{X}(P)$-computable in the generators $Y$ of $Q$.

For the proof of (5.2.1) we require a group $J=\left\langle y_{1}, y_{2}\right\rangle$ which is nilpotent of class 2 in which $\left|y_{1}\right|=\left|y_{2}\right|=\left|\left[y_{1}, y_{2}\right]\right|=n$. Thus $|J|=n^{3}$.

The generators $Y$ of $Q$ will be denoted (and specified later) by

$$
Y=\left\{w_{x} \mid x \in X\right\} \cup\left\{z_{x} \mid x \in X\right\} \cup J .
$$

Put $A=\operatorname{Dr}\left\{\left\langle z_{x}\right\rangle \mid x \in X\right\}$ (direct product); $Q$ will be a subgroup of $W=$ ( $P \mathrm{Wr} A$ ) wr $J$ where $P$ is embedded in the base group of $P \mathrm{Wr} A$ diagonally and $P \mathrm{Wr} A$ is embedded in $(P \mathrm{Wr} A)^{J}=$ the base group of $W$, as the 1 -coordinate subgroup.

We now specify the generators of $Q$ introduced in (5.2.2).

(5.2.3) (i) $\left|z_{x}\right|=|x|$ for all $x \in X$; thus $\left|z_{x}\right|$ is $\omega_{X}(P)$-computable since $P$ is periodic. $A$ will be identified with the top group of the 1-coordinate of $W$. 
(ii) The element $w_{x}$ in the base group of $P \mathrm{Wr} A$ is defined by $w_{x}(t)=x^{-i}$ where $t \equiv z_{x}^{i}\left(\bmod \operatorname{Dr}\left\{\left\langle z_{\alpha}\right\rangle \mid x \neq \alpha \in X\right\}\right)$. The element $\left[w_{x}, z_{x}\right]$ is a diagonal element with value $x$; we henceforth write $\left[w_{x}, z_{x}\right]=x$ and this describes the diagonal embedding of $P$ in $P \mathrm{Wr} A$.

(iii) $J$ is the top group of $W$.

Note that $\left\langle w_{x} \mid x \in X\right\rangle$ is contained in the set $\Lambda$ of all elements in the base group of $P \mathrm{Wr} A$ which have finite range. The set $\Lambda$ is obviously a normal subgroup of $P \mathrm{Wr} A$ and as $P$ is periodic, an element of $\Lambda$ must have finite order. Thus, $\left\langle w_{x} \mid x \in X\right\rangle^{A}$ is a periodic $\pi$-group and it now follows easily that $Q=\langle Y\rangle$ is a periodic $\pi$-group.

Also, $\omega_{X}(P) \leqslant \omega_{Y}(Q)$ since $x=\left[w_{x}, z_{x}\right]$. For the next part of the proof of Step 1 we verify

$$
\omega(P, Q) \leqslant \omega_{Y}(Q)=\omega_{X}(P) .
$$

Let $h \in Q=\langle Y\rangle$; since we already know that $\omega_{X}(P) \leqslant \omega_{Y}(Q)$, (5.2.4) will follow if we specify an algorithm effective in $\omega_{X}(P)$ to decide whether or not $h \in P$ and, if so whether or not $h=1$. First reduce $h$ to the form $h=\sigma\left(\prod_{g \in J} f_{g}^{g}\right)$ where $f_{g} \in\left\langle w_{x}, z_{x} \mid x \in X\right\rangle \leqslant P \operatorname{Wr} A$ and $\sigma \in J$. If $\sigma \neq 1$ we conclude that $h \notin P$, so assume $\sigma=1$. Note that the factors $\left\{f_{g}^{g} \mid g \in J\right\}$ commute pairwise since $P \operatorname{Wr} A$ is embedded as the 1-coordinate subgroup of the base group of $W$. Next we reduce every $f_{g}, g \in J$ :

$$
f_{g}=a_{g}\left[\prod_{i=1}^{n}\left(\left(w_{x_{i}}\right)^{k_{i}}\right)^{u_{i}}\right]
$$

where $a_{g}, u_{i} \in A, x_{i} \in X$ and $k_{i}$ is an integer (all of which depend on $g$ ). Using a $\omega_{X}(P)$-oracle to compute the orders of the $z_{x}$ we can determine for all $g \in J$ whether or not $a_{g}=1$. If $a_{g} \neq 1$ for some $g \in J$ then we conclude $h \notin P$. So, we can assume that $a_{g}=1$ and so $f_{g}$ is in the base group of $P \mathrm{Wr} A$ for all $g \in J$.

We will now show how to decide, for every $g \in J$, (i) whether $f_{g}$ lies in the diagonal of $P^{A}$ and (ii) if so, whether $f_{g}=1$. Having decided (i) and (ii) we decide whether $h \in P$ as follows. If $f_{g} \neq 1$ for some $g \neq 1$ then $h \notin P$ since $P$ is embedded in the 1-coordinate of $W$; if $f_{g}=1$ for all $g \neq 1$ then $h \in P$ if and only if $f_{1}$ is in the diagonal of $P$ Wr $A$ and $h=1$ if and only if $f_{1}=1$.

To decide (i) and (ii) let

$$
f_{g}=f=\prod_{i=1}^{n}\left(\left(w_{x_{i}}\right)^{k_{i}}\right)^{u_{i}}
$$

as in (5.2.5) with $a_{g}=1$. Let $z_{1}, \ldots, z_{j}$ denote all of the generators of $A$ which occur in the elements $u_{1}, \ldots, u_{n}$ and let $A_{0}=\left\langle z_{1}, \ldots, z_{j}, z_{x_{1}}, \ldots, z_{x_{n}}\right\rangle$. Thus, $A_{0}$ is a finite group and the computation of the values of $f$ is identical on the cosets of $A_{0}$ in $A$; i.e., if $a \in A_{0}$ and $t \in \operatorname{Dr}\left\{\left\langle z_{x}\right\rangle \mid z_{x}\right.$ is not one of the generators of $\left.A_{0}\right\}$, then $f(t+a)=f(a)$. Thus, to check whether $f$ is diagonal (resp., nontrivial) we need only compute its values on $A_{0}$ using a decision procedure for $\omega_{X}(P)$ to get the orders of the $z_{x}$ 's and to compare the values of $f$ that we obtain. These values are obtained from conjugates of the $w_{x}$, and so are computed as words in the generators $X$ of $P$. This completes the proof of (5.2.4). 
The final part of Step 1 is the proof of (5.2.1) which we now take up.

In the group $J$ we let $y=\left[y_{1}, y_{2}\right]$. Then $|y|=n>2$. If $x \in X$, then

$$
x=\left[w_{x}, z_{x}\right]=\left[\left[w_{x}^{-1}, y\right],\left[z_{x}^{-1}, y^{2}\right]\right]
$$

because $y^{2} \neq 1$ and the embedding of $P \operatorname{Wr} A$ into $W$ is on the 1-coordinate. Since $y$ is the commutator $\left[y_{1}, y_{2}\right]$ whose entries have order $n$ we have

$$
\left[w_{x}^{-1}, y\right]=\left(y^{-1}\right)^{w_{x}^{-1}} y=c_{1} c_{2},
$$

a product of two such commutators. Similarly, noting that $y^{2}=\left[y_{1}^{2}, y_{2}\right]$ we have $\left[z_{x}^{-1}, y^{2}\right]=c_{3} c_{4}$. The computation from $x$ of the entries of the commutators $c_{i}$ (the $d_{i}$ 's and $e_{i}$ 's) in the generators $Y$ of $Q$ is clearly effective. This concludes the proof of Step 1.

Step 2. We now embed $P=\langle X\rangle \tau$-effectively into a $(2 n+1)$-generator periodic $\pi$-group $R$.

Let $H=\left\langle h_{1}, \ldots, h_{n}\right\rangle$ be an infinite finitely generated $\pi$-group with $\omega(H) \leqslant \tau$ as given in the hypothesis of Theorem 2. Thus, $H$ does not have exponent 2 and $B=H \mathrm{wr} H$ has $2 n$ generators as in (5.1.1) and $\omega(B)=\omega(H)$.

Let $M$ be the sparse subset of $B$ as given in (5.1.2); from (5.1.2)(ii), $M$ is recursive in $\omega(B) \leqslant \tau$. Let $x \in X$ and for $1 \leqslant i \leqslant 8$ define $\alpha_{i}(x)$ by

$$
\alpha_{i}(x)= \begin{cases}d_{i} & \text { if } 1 \leqslant i \leqslant 4 \\ e_{i} & \text { if } 5 \leqslant i \leqslant 8\end{cases}
$$

where the $d_{i}$ and $e_{i}$ 's are as in (5.2.1). As noted earlier $\alpha_{i}(x)$ is effectively computable as a word in the generators $Y$ of $Q$ from $x$ and $i$. Now let $\left\{x_{j} \mid j \geqslant 0\right\}$ be an effective enumeration of the recursive set $X$ (if $X$ is finite, put $x_{j}=1$ after a point). We now define an element $v$ in the base group of $V=Q \mathrm{Wr} B$ as follows:

$$
v(c)= \begin{cases}1 & \text { if } c \notin M, \\ \alpha_{i}\left(x_{j}\right) & \text { if } c=t_{8 j+i}(1 \leqslant i \leqslant 8) .\end{cases}
$$

Thus given $c \in B$ we can compute the value $v(c) \in Q \tau$-effectively. Now put $R=v^{B} B \leqslant V$ and note that $R$ has $(2 n+1)$-generators, namely $v$ and the $2 n$ generators of $B$. We now show that $R$ has the properties asserted in Step 2 .

To prove that $R$ is a periodic $\pi$-group, note first that $|v|=n$ (since each of the $d_{i}$ and $e_{i}$ has order $n$ ); this together with the "sparseness" of $M$ implies that $v^{B}$ is a periodic $\pi$-group [10, p. 247], and it is now immediate that $R$ is a periodic $\pi$-group. We now take up the embedding aspects of Step 2.

Define $\delta_{i} \in v^{B}$ by

$$
\delta_{i}=\left[v^{\left(t_{8_{j+i}}\right)^{-1}}, v^{\left(t_{8_{j+i+4}}\right)^{-1}}\right] \quad(j \geqslant 0,1 \leqslant i \leqslant 4)
$$

and notice that $\delta_{i}$ has support $M\left(t_{8_{j+}}\right)^{-1} \cap M\left(t_{8_{j+i+4}}\right)^{-1}=\{1\}$ by the sparseness of $M$. Further, for $1 \leqslant i \leqslant 4$ we have

$$
\begin{aligned}
\delta_{i}(1) & =\left[v\left(t_{8_{j+i}}\right), v\left(t_{8_{j+i+4}}\right)\right]=\left[\alpha_{i}\left(x_{j}\right), \alpha_{i+4}\left(x_{j}\right)\right] \\
& =\left[d_{i}, e_{i}\right]=c_{i} \quad\left(\text { corresponding to } x_{j} \text { in }(5.2 .1)\right) .
\end{aligned}
$$


Hence $\left[\delta_{1} \delta_{2}, \delta_{3} \delta_{4}\right](1)=x_{j}$, displaying the embedding of $P$ into the 1-coordinate of $v^{B}$. Since $M$ is $\tau$-recursive and $v$ is $\tau$-computable, the above embedding displays a $\tau$-effective procedure for computing the generators $x \in X$ in terms of the generators of $R$. Thus $\omega_{X}(P) \leqslant \omega(R)$.

Also since $\omega_{y}(Q)=\omega_{x}(P) \leqslant \tau$ and $v$ is $\tau$-computable, (5.1.3) together with Lemma 2 imply that $\omega(R) \leqslant \tau$ and that there is a $\tau$-effective procedure for deciding when an element in $v^{B}$ has support $\{1\}$. Now we use the effectiveness of $P$ in $Q(=$ the 1-coordinate of $V)$ to conclude that $\tau \geqslant \omega(P, R)$.

This concludes the proof of Step 2 and Theorem 2 now follows directly from Lemma 4 because the relation "is $\tau$-effectively embedded in" is transitive.

ADDED IN PROOF. We have recently seen the constructions of Grigorcuk for $p=2$ and of Gupta and Said for $p>2$ of Burnside groups which have solvable word problems. It follows that our Theorem 2 can be improved to read: Every countable $\pi$-group can be effectively embedded in a 2-generator $\pi$-group. In the proof we can choose the sparse subset $M$ to be recursive and it follows that in the definition of an effective embedding (Definition 1) we can strengthen condition (iii) by replacing "recursive in $\tau$ " by "recursive", which is a more natural concept.

\section{REFERENCES}

1. E. S. Golod, On nil-algebras and periodic groups, Izv. Akad. Nauk SSSR Ser. Mat. 28 (1964), 273-276; English transl. in Amer. Math. Soc. Transl. (2) 48 (1965), 102-106.

2. P. Hall, Finiteness conditions for soluble groups, Proc. London Math. Soc. (3) 4 (1954), 419-436.

3. K. K. Hickin, Effective embeddings of residually finite groups (in preparation).

4. K. K. Hickin and R. E. Phillips, Some isomorphism types of 2-generator subgroups in small varieties (in preparation).

5. Non-isomorphic Burnside groups of exponent $p^{2}$, Canad. J. Math. 30 (1978), 180-189.

6. S. Jeanes, Counting the periodic groups generated by two finite groups, Bull. London Math. Soc. (2) 22 (1979), 195-199.

7. R. Lyndon and P. Schupp, Combinatorial group theory, Springer-Verlag, New York, 1977.

8. B. H. Neumann and H. Neumann, Embedding theorems for groups, J. London Math. Soc. 34 (1959), 465-479.

9. P. S. Novikov and S. I. Adyan, Infinite periodic groups, Izv. Akad. Nauk SSSR Ser. Mat. 32 (1968), 212-244, 251-524, 709-731; English transl. in Math. USSR-Izv. 2 (1968), 209-236, 241-479, 665-685.

10. R. E. Phillips, Embedding methods for periodic groups, Proc. London Math. Soc. (3) 35 (1977), $238-256$.

11. G. E. Sacks, Degrees of unsolvability, Ann. of Math. Studies, No. 55, Princeton Univ. Press, Princeton, N. J., 1963.

12. J. S. Wilson, Embedding theorems for residually finite groups, Math. Z. 174 (1980), 149-157.

13. K. K. Hickin, Isomorphism types of center-by-metabelian groups (in preparation).

Department of Mathematics, Michigan State University, East Lansing, Michigan 48824 